\title{
Transplantes y Pueblos Indígenas: un Enfoque Inicial
}

\author{
Estevão Rafael Fernandes ${ }^{1} \stackrel{D}{ }$, Ana Karoline Nóbrega Cavalcanti ${ }^{2}$
}

1. Universidade Federal de Rondônia RóR Porto Velho (RO), Brasil.

2. Secretaria de Estado de Saúde - Porto Velho (RO), Brasil.

\section{doi https://doi.org/10.53855/bjt.v25i1.439_esp}

Autor correspondiente:

estevaofernandes@gmail.com

Editor de Sección

Ilka Boin

Recibido

Ene. 12, 2022

Aprobado

Ene. 13, 2022

Conflicto de interés

Nada a declarar

Cómo Citar

FernandesER,CavalcantiAKN.Transplantes

y Pueblos Indígenas: un Enfoque Inicial.

BJT. 2022; 25(01): e0122. https://doi.org/10.

53855/bjt.v25i1.439_esp

eISSN

2764-1589

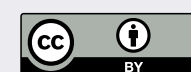

Resumen: Este artículo indica un conjunto de cuestiones relativas a los transplantes involucrando a personas indígenas. Se trata de un tema no abordado en la literatura del país y para el cual es relevante dar un vistazo más atento, dados los aspectos no solamente médicos, sino también bioéticos, sociocosmológicos, religiosos, sociales y culturales. Desde una perspectiva interdisciplinaria, se intentó aquí llamar la atención para la importancia de tener en cuenta aspectos relativos a la constitución de la persona indígena; implicaciones referentes al consentimiento informado; y la necesidad de un enfoque sensible para las diferencias interculturales implicadas. La conclusión fue que se hace necesaria la formación de un protocolo en conjunto con organizaciones indígenas y órganos oficiales que considere las especificidades culturales. Además de esto, un levantamiento tanto de las iniciativas institucionales en otros países como de la literatura sobre la temática aún es una tarea a ser hecha en Brasil.

Descriptores: Pueblos Indígenas; Salud de Poblaciones Indígenas; Obtención de Tejidos y Órganos.

\section{INTRODUCCIÓN}

Brasil es un país diverso étnicamente. En su territorio, se encuentran más de 300 etnias, hablando cerca de 280 lenguas diferentes. A pesar de que estos indígenas pueblen el imaginario como intocados, en medio de la Amazonía, aproximadamente el $40 \%$ de la populación indígena brasileña habita en ambientes urbanos, según estimativas del Instituto Brasileño de Geografía y Estadística. ${ }^{1}$

Desde el punto de vista sanitario, varios de estos pueblos ya enfrentan la llamada "transición epidemiológica", ${ }^{2}$ con un número creciente de muertes causadas por enfermedades crónicas - como obesidad, diabetes, problemas renales, problemas del corazón, por ejemplo -, consecuencia directa o indirecta del contacto con la sociedad envolvente. De la misma forma, problemas como falta de saneamiento, contaminación proveniente de minas o contacto directo con agrotóxicos ${ }^{3}$ afectan cotidiana y sistemáticamente a comunidades indígenas, incluyendo pueblos aislados. En este contexto, se puede afirmar ser cuestión de tiempo para haber una demanda creciente por transplantes por parte de esta población, algo hasta aquí poco enfrentado tanto por la literatura del país como por gestores y legisladores.

Ya no se trata de considerar a los pueblos indígenas en territorio nacional como ajenos a las demandas y a los problemas más generales de la población como un todo, sino, al contrario, de buscar extender a ellos toda la asistencia a la cual tienen derecho, siempre que sean respetados aspectos específicos de sus culturas. 


\section{DISCUSIÓN}

Estas consideraciones iniciales llevan a una pregunta: ¿qué cuestiones deben ser tenidas en cuenta en los trasplantes involucrando a pueblos indígenas?

Dados los límites de este texto, pensamos ser importante destacar sobre todo tres aspectos ante este problema. En primer lugar, se tienen las especificidades al trabajar en otra perspectiva de persona. Dicho esto, brevemente - este punto será desarrollado más adelante -, en el Occidente la noción de persona se confunde con la de Homo sapiens. En los pueblos indígenas, por otro lado, como las formas de clasificación del mundo son diversas y no se fundamentan en nuestros presupuestos biologizantes, un sujeto se convierte en persona por intermedio de una serie de complejas relaciones sociales y rituales. El cuerpo no es, en estas sociedades, mero artefacto físico, sino la suma de relaciones sociales y cosmológicas comprendidas tan solamente con base en sus culturas.

En segundo lugar, se hace necesario indicar implicaciones prácticas con respecto al consentimiento informado: ¿cómo obtenerlo de la mejor forma posible, en un contexto intercultural?

Finalmente, se intentó llamar la atención para la importancia de un enfoque sensible para estas diferencias culturales y para un presupuesto significativo tratándose de este tipo de problemática: la intervención en un cuerpo indígena repercute en toda su comunidad de varias maneras diferentes, y, en lo que se refiere a transplantes, esta intervención se complejiza considerablemente. Se concluye aquí ser relevante la constitución, en conjunto con organizaciones indígenas y órganos indigenistas, de un protocolo que regule transplantes en contextos interculturales, como modo de resguardar tanto comunidades indígenas como equipos médicos por todo el país.

Hechas estas consideraciones, es interesante indicar cómo la problemática transplantes involucrando a personas indígenas no causa alerta entre investigadores en Brasil. Buscándose en las principales bases de datos del área de la salud (PubMed, Literatura Latinoamericana y del Caribe en Ciencias de la Salud - Lilacs, Biblioteca Electrónica Científica Online - SciELO, entre otras), por medio de descriptores tales como indígenas o pueblos indígenas y transplante, lo que se vio fue la completa falta de textos producidos sobre el tema - incluso en referencias importantes acerca de salud indígena en el país. ${ }^{4}$ Además de esto, aunque dispongamos de una política relativamente avanzada dirigiendo el Subsistema de Atención a la Salud Indígena, es importante indicar que la Política Nacional de Atención a la Salud de los Pueblos Indígenas, aprobada en el 2002, no hace mención a la palabra transplante ni siquiera una vez en todo su texto.

Este vacío al respecto del tema en el contexto brasileño llama la atención en relación a lo que se produce fuera del país, en la academia o más allá de ella. Una rápida búsqueda usándose los mismos descriptores lleva a varios textos sobre el tema en diferentes contextos nacionales y culturales - algunos ejemplos dejan esta diversidad clara. ${ }^{5-11}$

Ya institucionalmente, hay varias iniciativas oficiales exitosas siendo desarrolladas o en curso en otros países en el sentido de pensar transplantes en pueblos indígenas. Basta decir que el Office of Minority Health del U.S. Department of Health and Human Services - el equivalente a nuestro Ministerio de la Salud - posee un websitio específico sobre donación de órganos para nativos americanos de los Estados Unidos continental y de Alaska. ${ }^{12}$ En aquel país, conforme las informaciones publicadas en el sitio web, fueron realizados 280 transplantes involucrando a personas indígenas en el 2020, cuando había más de 900 americanos nativos esperando transplantes de riñones, hígado, pulmón y corazón.

En Canadá, a su vez, hay aplicaciones de celular dirigidas para las personas pertenecientes a first nations, como Kidney Check Program, o iniciativas para discutir cómo el sistema de salud puede acoger pacientes indígenas y sus familias en las acciones referentes a transplantes de órganos - como en el encuentro virtual ocurrido en diciembre del 2021, promovido en colaboración con Network Environments for Indigenous Health Research National Coordinating Centre, del First Nations and Métis Organ Donation and Transplantation Network y de Can-SOLVE CKD Network (Fig. 1).

No se dispone aquí de espacio para analizar, de forma detenida y merecida, las diferencias entre las políticas indigenistas en estos contextos nacionales - se espera hacerlo en el futuro, así como un ejercicio de revisión de esta literatura. Basta, aquí, apuntar de modo preliminar que tal sensibilidad es fruto no solamente de un largo trayecto de demandas de los movimientos indígenas organizados en estos países, sino también de una relación diferente en términos institucionales con tales pueblos por parte de esos Estados y construida históricamente. ${ }^{14}$

Sin embargo, hay un punto específico que interesa directamente a quien busca pensar transplantes y pueblos indígenas desde una perspectiva legal. En Brasil, tenemos una legislación indigenista nacida en pleno régimen militar - el Estatuto del Indio es de 1973 - basada en la idea de tutela. El objetivo era "integrar a los indios pacíficamente a la Unión Nacional”, ejerciendo sobre estas comunidades todo el control posible, sobre todo a la luz de la Doctrina de Seguridad Nacional. ${ }^{15}$ El concepto de una autonomía cultural solamente vino a ser respetado con la Constitución Federal de 1988, pero, aún así, el respeto a las culturas indígenas, previsto en el Artículo 231 de la Carta Constitucional, queda sometido, hasta los días de hoy, al escrutinio del órgano tutor - o sea, la Fundación Nacional del Indio (Funai). En suma, esto significa que cualquier intervención, como un transplante, por ejemplo, dada la falta de una previsión legal específica o de protocolos constituidos de forma clara y colaborativa, puede transformarse fácilmente en un campo de batalla burocrático y jurídico involucrando a Funai, Abogacía General de la Unión, Ministerios de la Salud, de los Derechos Humanos, de la Justicia, Público, organizaciones indígenas, entre otros. La 


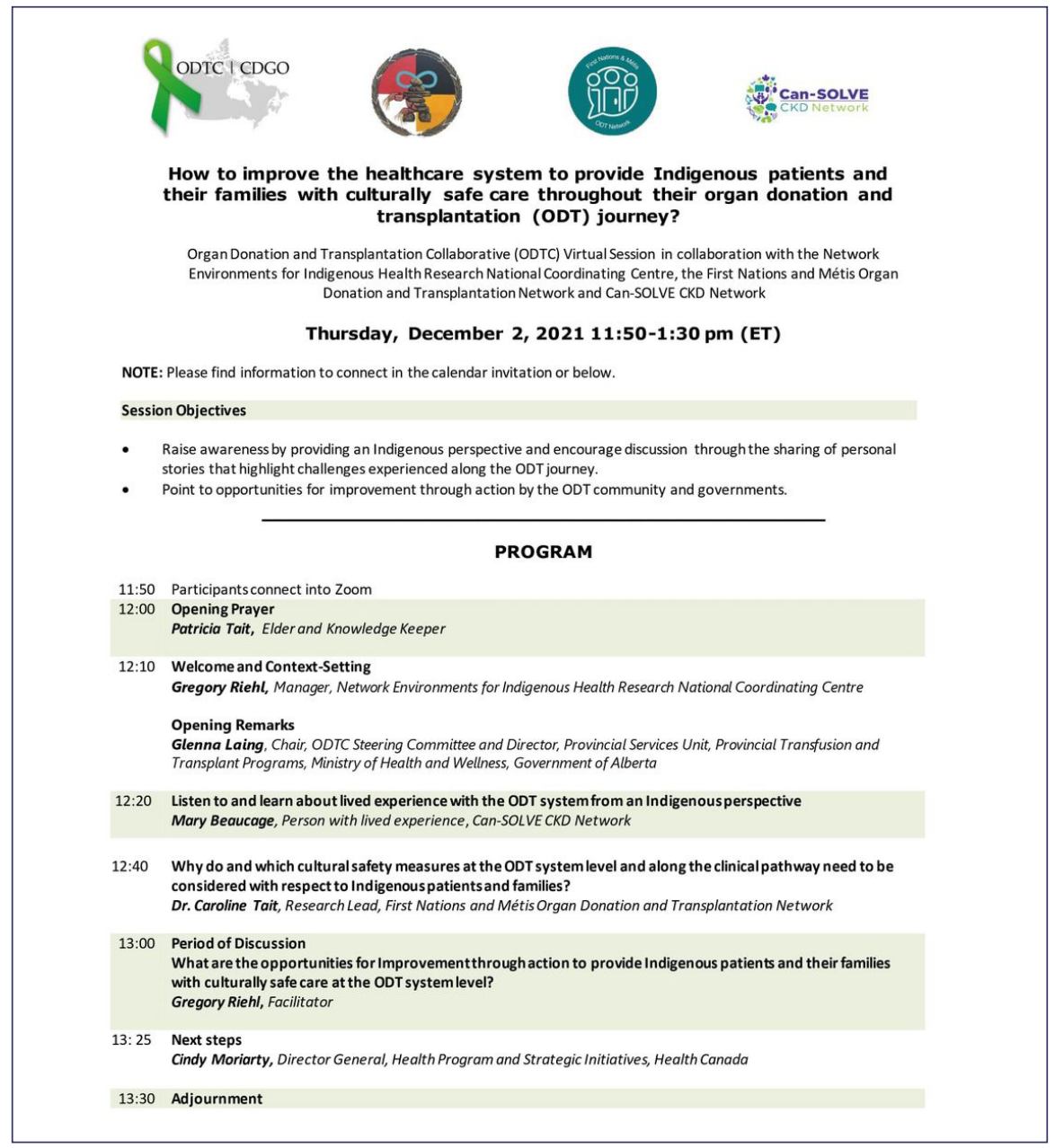

Fuente: First Nations and Métis Organ Donation and Transplantation Network.13

Figura 1. Organ donation and transplantation collaborative, Canadá.

idea de un protocolo vendría no solamente a respaldar a los equipos de salud, sino también a resguardar la cultura de estos pueblos y sus propias nociones de salud, enfermedad y persona.

No se trata, es importante destacarlo, tan solamente de respetar las culturas indígenas - o sea, un presupuesto moral, sino también de una norma establecida desde la firma de la Convención 169 de la Organización Internacional del Trabajo (OIT), transformada en ley en Brasil en el 2004, firmando la obligatoriedad de la consulta previa e informada a los pueblos indígenas sobre decisiones que impacten directamente sus vidas.

El punto aquí es: ¿de qué manera un transplante, por ejemplo, impactaría a estas sociedades ante la perspectiva de sus culturas y cómo mitigar estos impactos?

Como fue dicho anteriormente, la noción de persona entre los pueblos indígenas es un bueno punto de partida para comprender este tipo de problema. La cuestión es investigada en el ámbito de la antropología brasileña hace más de 40 anos ${ }^{16}$ y puede ser resumida de la siguiente forma: el cuerpo amerindio está construido por un conjunto de relaciones simbólicas, rituales, de parentesco, de nominación. $\mathrm{O}$ sea, el sujeto se convierte en un lugar de encuentro de diversas relaciones sociales, siendo construido y comprendido por las categorías de pensamiento nativas. Lo que los occidentales entienden como individuo en los pueblos indígenas da lugar a una unidad social que opera con relación a las redes de parentesco, mitologías, cosmologías y perspectivas nativas de naturaleza, por ejemplo. Una vez que el sujeto es este cruzamiento de cadenas simbólicas, él debe ser comprendido con base en ellas, de tal modo que una acción sobre el cuerpo nativo es, en regla, algo que afecta a todo el corpus de aquella sociedad.

¿Cómo esto funciona en la práctica? Uno de los autores de este texto tuvo la oportunidad de analizar, hace algunos años, ${ }^{17}$ ideologías en torno de la concepción de persona entre los indios Yê de Brasil Central, permitiendo dar un ejemplo de cómo operan estas concepciones en estos pueblos. En resumen, en algunas sociedades, el feto es visto como sustancia relacionada directamente al padre y a sus parientes consanguíneos, de tal forma que cualquier alimento ingerido por su padre, tíos, abuelo paterno y primos paralelos repercute en el cuerpo del niño por varios años después del nacimiento. Esto hace que haya una serie de restricciones y tabúes alimentarios por parte de estas 
personas, a fin de resguardar su salud: si un tío se alimenta de carne de armadillo, el niño comerá tierra; en el caso que su padre coma frijoles, su piel se pondrá negra, etc. Es frecuente, por ejemplo, buscar un culpado por romper estos tabúes en el caso de enfermedades más graves, para restablecer su salud. Niños en estas sociedades pasan años sin un nombre propio, pues deben esperar a que sus cuerpos tengan fuerza suficiente para aguantar un nombre - o sea, tener relativa autonomía como sujetos -, y aún así hay varios cambios de nombre a lo largo de la vida, marcando modificaciones importantes en el status de estas personas: nuevos hitos en sus cuerpos, en los rituales de los cuales participan, de los papeles que ocupan en sus comunidades, entre otros.

Otro ejemplo también muy conocido y bien documentado se refiere a las muestras de sangre de los indígenas Yanomami (Roraima/Amazonas) llevadas por investigadores de los Estados Unidos en los años 1960 y 70 . Por más de cuatro décadas, los liderazgos Yanomami buscaron recuperar estas muestras a fin de aplacar los espíritus de aquellas personas ya fallecidas cuya sangre fue retirada. En aquella cultura, los muertos deben ser cremados en una serie de complejos rituales, y la sangre congelada en alguna universidad impedía tal ceremonia, una vez que parte de aquellas personas no había pasado por estos ritos.

Ejemplos en este sentido son muchos e indican lo que se ha intentado apuntar aquí: procesos de salud, enfermedad, corporalidad, cura y cuidados entre pueblos indígenas, de modo general, son parte relevante de la vida colectiva de estas sociedades y solamente pueden ser comprendidos a la luz de sus culturas. Por ser la persona indígena una constitución social, estas intervenciones poseen también repercusión social.

Esto lleva a otro punto: las repercusiones bioéticas de estas cuestiones en la práctica. Mucho se conoce sobre la Resolución del Consejo Nacional de Salud (CNS) n 466/2012, la cual establece las directrices para investigaciones en seres humanos, pero se llama la atención aquí para otras dos normas, relativamente poco conocidas. La primera, n 304/2000, aún en vigor (incluso habiendo sido hecha bajo vigencia de la Resolución no 196/1996), se refiere a la ética en investigación específicamente con pueblos indígenas. La segunda norma es la Resolución CNS no 510/2016, que normaliza las ciencias humanas en las investigaciones que puedan acarrear riesgos para la vida cotidiana.

Estas dos normas son traídas aquí por dar algunas directrices importantes en lo referente a enfoques posibles en la búsqueda por consentimiento, en el caso de transplante involucrando a personas indígenas. Tales directrices son sugerencias, pudiendo ser readecuadas, siendo lo ideal-se repite - la creación de un protocolo específico dirigido para estas poblaciones. Cuestiones importantes tales como el respeto a las particularidades culturales y a las esferas de decisión nativas se encuentran bien establecidas, pero falta, por ejemplo, la previsión en el uso de intérpretes o traductores para auxiliar en aquellos procedimientos cuyos involucrados no posean conocimiento pleno de la lengua portuguesa. Por más que parezca preciosismo, a título de comparación, algunas comisarías de atención a la mujer del país poseen mujeres intérpretes a fin de auxiliar en el acogimiento de las indígenas víctimas de violencia. A fin de cuentas, incluso aquellas que viven cercanas a núcleos urbanos no necesariamente logran comunicarse de manera integral en portugués.

\section{CONCLUSIÓN}

Se vio que el transplante involucrando a pueblos indígenas en Brasil es aún algo a ser enfrentado tanto por legisladores como por gestores e investigadores. Aunque haya una acumulación de literatura y algunas iniciativas en otros países en este sentido, el tema no ha suscitado mayores reflexiones en tierras brasileñas. Este artículo es un primer paso en esta dirección. Una de nuestras hipótesis es que la transición epidemiológica registrada en varias de estas comunidades y problemas sociales y ambientales diversos hará surgir, brevemente, una demanda por transplantes por parte de estos pueblos, como ocurre en otros países.

A lo largo de este texto, se intentó mostrar, aunque sea resumidamente, cómo las cuestiones culturales poseen implicaciones directas al pensar el transplante en una perspectiva intercultural: corporalidad y persona amerindias son parte de un conjunto complejo de relaciones sociales y culturales las cuales deben ser respetadas y consideradas en este tipo de intervención. No obstante, dada esta complejidad, se sugiere aquí la construcción, en colaboración con organizaciones indígenas y órganos de Estado, de un protocolo de transplantes que tenga en cuenta estas especificidades.

Además de esto, parece urgente la tarea de realizar un levantamiento detallado de la literatura sobre transplantes en pueblos indígenas en otros contextos nacionales, así como de las iniciativas ejecutadas en otros países, para aprender con estas experiencias.

\section{CONTRIBUCIÓN DE LOS AUTORES}

Contribuciones científicas e intelectuales sustantivas al estudio: Fernandes ER and Cavalcanti AKN; Concepción: Fernandes ER and Cavalcanti AKN. 


\section{DISPONIBILIDAD DE DATOS DE INVESTIGACIÓN}

Todos los datos fueron generados o analizados en el presente estudio.

\section{FINANCIAMIENTO}

No se aplica.

\section{AGRADECIMIENTOS}

No se aplica.

\section{REFERENCIAS}

1. Instituto Brasileiro de Geografia e Estatística (IBGE). Indígenas. Gráficos e tabelas [Internet]. Brasil: IBGE [accedido en 17 ene. 2022 ]. Disponible en: https://indigenas.ibge.gov.br/graficos-e-tabelas-2.html

2. Coimbra Jr. CE, Flowers NM, Salzano F, Santos RV. Xavante in transition: health, ecology and bioanthropology in Central Brazil. Ann Arbor: University of Michigan Press; 2002.

3. Gonçalves GMS, Gurgel IGD, Costa AM, Almeida LR, Lima TFP, Silva E. Uso de agrotóxicos e a relação com a saúde na etnia Xukuru do Ororubá, Pernambuco, Brasil. Saúde Soc. 2012;21(4):1001-12. https://doi.org/10.1590/S0104-12902012000400017

4. Buchillet D. Bibliografia crítica da saúde indígena no Brasil (1844-2006). Quito: Abya-Yala; 2007.

5. Howson P, Irish AB, D’orsogna L, Chakera A, Swaminathan R, Perry G, et al. Allograft and Patient Outcomes Between Indigenous and Nonindigenous Kidney Transplant Recipients. Transplantation. 2020;104(4):847-55. https://doi.org/10.1097/TP.0000000000002891

6. Barraclough KA, Grace BS, Lawton PMSP, McDonald SP. Residential location and kidney transplant outcomes in indigenous compared with nonindigenous Australians. Transplantation. 2016;100(10):2168-76. https://doi.org/10.1097/TP.0000000000001007

7. Keddis MT, Sharma A, Ilyas M, Zhang N, Khamash H, Leischow SJ, et al. Transplant center assessment of the inequity in the kidney transplant process and outcomes for the Indigenous American patients. PLoS One. 2018;13(11):e0207819. https://doi.org/10.1371/ journal.pone.0207819

8. Khanal N, Lawton PD, Cass A, McDonald SP. Disparity of access to kidney transplantation by Indigenous and non-Indigenous Australians. Med J Aust. 2018;209(6):261-6. https://doi.org/10.5694/mja18.00304

9. Walker RC, Abel S, Reynolds A, Palmer SC, Walker C, Tipene-Leach DC. Experiences, perspectives and values of Indigenous peoples regarding kidney transplantation: systematic review and thematic synthesis of qualitative studies. Int J Equity Health. 2019;18:204. https://doi.org/10.1186/s12939-019-1115-y

10. Majoni SW, Ullah S, Collett J, Hughes JT, McDonald S. Weight change trajectories in Aboriginal and Torres Strait islander Australians after kidney transplantation: a cohort analysis using the Australia and New Zealand Dialysis and Transplant registry (ANZDATA). BMC Nephrol. 2019;20:232. https://doi.org/10.1186/s12882-019-1411-1

11. Boan P, Swaminathan R, Irish A. Infectious complications in indigenous renal transplant recipients in Western Australia. Intern Med J. 2017;47(6):648-55. https://doi.org/10.1111/imj.13450

12. U.S. Departament of Health and Human Services. Organ Donation and American Indians/Alaska Natives. Estados Unidos: U.S. Departament of Health and Human Services, Office of Minority Health; 2021.

13. First Nations and Métis Organ Donation and Transplantation Network. Facebook [Internet]. [acedido en ene. 2022]. Disponible en: https://www.facebook.com/pg/FirstNationsMetisODT

14. Fernandes ER. Descolonizando sexualidades: enquadramentos coloniais e homossexualidade indígena no Brasil e nos Estados Unidos. Brasília: Editora Universidade de Brasília; 2020. https://doi.org/10.26512/9786558460442

15. Lima ACS. Um grande cerco de paz: poder tutelar, indianidade e formação do Estado no Brasil. Petrópolis: Vozes; 1995.

16. Seeger A, Matta R, Viveiros de Castro E. A construção da pessoa nas sociedades indígenas brasileiras. Boletim do Museu Nacional. 1979:2-19. Série Antropologia.

17. Fernandes ER. Do Tsihuri ao Waradzu: o que as ideologias xavante de concepção, substância e formação da pessoa nos dizem sobre o estatuto ontológico do outro? Horiz Antropol. 2010;16(34):453-77. https://doi.org/10.1590/S010471832010000200019 